\title{
Improved Sparse Representation Super-Resolution algorithm for Remote Sensing Image
}

\author{
Zhu Fuzhen ${ }^{1, a}$, Huang Xin ${ }^{1}$, Liu Yue ${ }^{1}$ and Zhu Haitao ${ }^{2}$ \\ ${ }^{1}$ College of Electronic Engineering, Heilongjiang University, Harbin, 150080, China; \\ ${ }^{2}$ Heilongjiang Duobaoshan Copper Industry lnc. , Heihe, 164300, China
}

\begin{abstract}
In order to obtain higher quality super-resolution reconstruction (SRR) of remote sensing images, an improved sparse representation remote sensing images SRR method is proposed in this paper. First, lowresolution image is processed by improved feature extract operator. The high-resolution image and lowresolution image blocks have the same sparse representation coefficient, so the SRR image with higher spatial resolution can be derived from the sparse representation coefficients which have been obtained from low-resolution image. The improved feature extraction operator is a method to get more detail and texture information from the training images. Experiment results show that more texture details can be obtained in the result of SRR remote sensing images subjectively. At the same time, the objective evaluation parameters are improved greatly. The peak PSNR is increased about $2.50 \mathrm{~dB}$ and $0.50 \mathrm{~dB}$, RMSE is decreased about 2.80 and 0.3 compared with bicubic interpolation algorithm and Ref[8] algorithm respectively.
\end{abstract}

\section{Introduction}

Image super-resolution reconstruction (SRR) is defined as a mapping relationship between high-resolution images (HRI) and low-resolution images (LRI) by fusing information in multiple frames LRIs ${ }^{[1-3]}$. The process of SRR is done based on reasonable assumptions model or prior knowledge to recover HRI. It has been used widely in the fields of remote sensing image, medical ultrasound imaging, video surveillance, and so on. Currently, SRR algorithms mainly include three algorithms, that is interpolation-based SRR, reconstruction-based SRR and learning-based SRR ${ }^{[4]}$. Recently, the learning-based SRR algorithm has been a popular research direction in the field of SRR, in which mapping relationship between HRI and LRI can be obtained by training remote sensing images in a library, and then HR image can be reconstructed from the mapping matrix. In 2002, Freeman $^{[5]}$ proposed an example-based learning SRR algorithm to face images, in which the LR to HR prediction is learned in Markov Random Field (MRF) by back propagation. Liu ${ }^{[6]}$ proposed a two-step statistical method based on local and global Principal Component Analysis (PCA) model for face images SRR. Although the algorithm produced good results, the overall PCA model often produced mean face image effect. As a result, probable partial block model is complex and computation demanding is large. Chang ${ }^{[7]}$ applied the idea of neighborhood embedding to learning-based SRR, which greatly reduced the number of training samples, but needed to artificially select the number of neighbors, so it is easier to produce over-fitting. In 2010, Yang ${ }^{[8]}$ proposed sparse representation SRR by reconstructing HRIs corresponding to LRIs. In this method, a pair of HRI and LRI dictionaries is obtained by training a large number of image blocks, instead of using the image block pairs sampled in HRIs directly. Therefore, costing time of this algorithm is reduced effectively. At the same time, ability of generation and anti-noise are improved greatly. In this paper, feature extraction is improved and the reconstructed image quality and visual effect is improved compared with Yang's method.

\section{Remote sensing image sparse representation}

LRIs $X_{l}$ can be obtained from HRIs $Y_{h}$ after blurring and down-sampling [9]. This process is called image degradation, and which is often modeled as equation (1):

$$
X_{l}=S H Y_{h}+n
$$

Where $S$ is down-sampling matrix, $H$ is a fuzzy degradation matrix, $n$ is all external noise.

In this paper, the methods in reference(Ref) [8] are mainly applied to block image processing. In order to avoid the complex calculations that caused by different sizes $X_{l}$ and $Y_{h}$, the LRI with the same size of $Y_{h}$ was obtained through interpolation and magnification of $X_{l}$. As showed in equation (2):

$$
X_{l}^{\prime}=I S H Y_{h}+I n=L Y_{n}+n^{\prime}
$$

a Corresponding author: Email: zhufuzhen@hlju.edu.cn.

Zhu Fuzhen (1978-), doctor, master supervisor, research interests : image super-resolution, compressive sensing, neural network, deep learning, et al. 
where, $I$ represent the interpolation amplification operation, $L=I S H, \quad n^{\prime}=I n$.

It is assumed that the HR image block $y_{h}$ can be linearly represented by the column vector of the HR image block dictionary $D_{h} \in R^{m \times k}(m \ll k)$. As shown in equation (3):

$$
y_{h}=D_{h} \alpha \text { s.t }\|\alpha\|_{0} \ll k
$$

where, $\alpha$ is a sparse representation coefficient, $\alpha \in R^{k}$, $\|*\|_{0}$ is the number of non-zero elements in a vector and $D_{h}$ is a dictionary trained by HRIs $Y_{h}$. Then LRI block $x_{l}^{\prime}$ is jointly obtained by formulas (2) and (3).

$$
x_{l}^{\prime}=L y_{h}+n^{\prime}=L D_{h} \alpha+n^{\prime}
$$

Equation (4) can be expressed as

$$
x_{l}^{\prime}=D_{l} \alpha+n^{\prime}
$$

where $D_{l}=L D_{h}, D_{l}$ is the LRI block dictionary. $x_{l}^{\prime}=D_{l} \alpha=n^{\prime}$.

$$
\left\|x_{l}^{\prime}-D_{l} \alpha\right\|_{2} \leq \varepsilon
$$

where, $\varepsilon$ is related to the noise energy of $n^{\prime}$. It can be seen from the above deduction that within the error $\mathcal{E}$, $y_{h}$ and $x_{l}^{\prime}$ have the same $\alpha$ in their corresponding dictionaries $D_{h}$ and $D_{l}$. Therefore, $y_{h}$ can be found by solving $\alpha$ under its dictionary $D_{l}$.

In practice, thorough sparseness is impossible, so equation (5) needs to be optimized and converted into Lagrange form ${ }^{[10]}$.

$$
\min _{\alpha}\left\|F x_{l}^{\prime}-D_{l} \alpha\right\|_{2}^{2}+\lambda\|\alpha\|_{1}
$$

where $\lambda$ is a regularization parameter, and $F$ is a feature extraction operator. The training sample of $D_{l}$ is extracted from the features of $X_{l}^{\prime}$.

\section{Dictionary training and learning}

In this paper, joint training dictionary algorithm in ref[8] is used to train HR and LR image samples, and high-low resolution dictionary $D_{h}$ and $D_{l}$.are obtained respectively. Dictionary $D_{h}$ training process is as follows:

$$
\min _{D_{h}, A}\left\|Y_{h}-D_{h} A\right\|_{2}^{2} \text { s.t } \forall i,\left\|\alpha_{i}\right\|_{1}<t
$$

Where, $A$ represent a sparse matrix, $\alpha_{i} \in A$.

Dictionary $D_{l}$ is trained as equation(9):

$$
\min _{D_{l}, A}\left\|X_{l}^{\prime}-D_{l} A\right\|_{2}^{2} \text { s.t } \forall i,\left\|\alpha_{i}\right\|_{1}<t
$$

HRI $\left(y_{h}\right)$ and LRI $\left(x_{l}^{\prime}\right)$ have the same sparse coefficient $\alpha$, so the equation (8) and (9) can be combined as:

$$
\begin{gathered}
\min _{D_{l}, D_{h}, A} \frac{1}{N}\left\|Y_{h}-D_{h} A\right\|_{2}^{2}+\frac{2}{M}\left\|X_{l}^{\prime}-D_{l} A\right\|_{2}^{2} \\
\text { s.t } \forall i,\left\|\alpha_{i}\right\|_{1}<t
\end{gathered}
$$

Equation (10) is equivalent to

$$
\min _{D_{l}, D_{h}, A}\|Y-D A\|_{2}^{2} \text { s.t } \forall i,\left\|\alpha_{i}\right\|_{1}<t
$$

In formula (11)

$$
Y=\left[\begin{array}{l}
\frac{1}{\sqrt{N}} Y_{h} \\
\frac{1}{\sqrt{M}} X_{l}^{\prime}
\end{array}\right] D=\left[\begin{array}{l}
\frac{1}{\sqrt{N}} D_{h} \\
\frac{1}{\sqrt{M}} D_{l}
\end{array}\right]
$$

where, $N$ and $M$ are the dimensions of the sample image block vector form respectively. Dictionary $D_{h}$ and $D_{l}$ can be obtained by the sparse coding method ${ }^{[11]}$ through the combined input HR and LR sample images.

\section{Improvement of sparse representation remote sensing image SRR}

In Ref[8], sparse coefficient $\alpha$ is for each $x_{l}^{\prime}$ and gets $y_{h}$ by formula $y_{h}=D_{h} \alpha$. Sparse representation process can be transformed into the following optimization problem, as shown in (13):

$$
\min \|\alpha\|_{0}{ }_{\text {s.t }}\left\|F D_{l} \alpha-F x_{l}^{\prime}\right\|_{2}^{2} \leq t
$$

Feature extraction of $x_{l}^{\prime}$ can get its high frequency information, and the up-sampling of $x_{l}^{\prime}$ can be used as the low-frequency information of $y_{h}$, then the features of $x_{l}^{\prime}$ can be extracted as part of the sparse constraint.

In Ref[8], first-order and two order derivatives are used the characteristics of $x_{l}^{\prime}$. For an $5 \times 5$ image block, as showed in Figure 1:

Fig.1 Image block size of $5 \times 5$.

\begin{tabular}{|l|l|l|l|l|}
\hline$x_{1}$ & $x_{2}$ & $x_{3}$ & $x_{4}$ & $x_{5}$ \\
\hline$x_{6}$ & $x_{7}$ & $x_{8}$ & $x_{9}$ & $x_{10}$ \\
\hline$x_{11}$ & $x_{12}$ & $x_{13}$ & $x_{14}$ & $x_{15}$ \\
\hline$x_{16}$ & $x_{17}$ & $x_{18}$ & $x_{19}$ & $x_{20}$ \\
\hline$x_{21}$ & $x_{22}$ & $x_{23}$ & $x_{24}$ & $x_{25}$ \\
\hline
\end{tabular}

The first-order and second-gradient vectors of the center pixel of figure 1 are as follows:

$$
\begin{gathered}
\nabla x_{13}=\left[\begin{array}{l}
x_{14}-x_{13}+x_{13}-x_{12} \\
x_{18}-x_{13}+x_{13}-x_{8}
\end{array}\right]=\left[\begin{array}{l}
x_{14}-x_{12} \\
x_{18}-x_{8}
\end{array}\right] \\
\nabla^{2} x_{13}=\left[\begin{array}{l}
x_{15}-x_{13}-\left(x_{13}-x_{11}\right) \\
x_{23}-x_{13}-\left(x_{13}-x_{3}\right)
\end{array}\right] \\
=\left[\begin{array}{l}
x_{15}-2 x_{13}+x_{11} \\
x_{23}-2 x_{13}+x_{3}
\end{array}\right]
\end{gathered}
$$


The four one-dimensional filters are extracted ${ }^{[12]}$ by above formula, and then feature extract operators can be obtained as follows:

$$
\begin{gathered}
f_{1}=[-1,0,1] \quad f_{2}=f_{1}^{T} \\
f_{3}=[1,0,-2,0,1], \quad f_{4}=f_{3}^{T}
\end{gathered}
$$

LRI $x_{l}^{\prime}$ is processed by using $f_{1}, f_{2}, f_{3}$, and $f_{4}$ to obtain its high-frequency information. In this paper, the high-pass filter is improved as shown in equation (17).

$$
\begin{array}{cc}
f_{5}=[-2,1,0,-1,2] & f_{6}=f_{5}^{T} \\
f_{7}=[2,-2,0,-2,2] & f_{8}=f_{7}^{T}
\end{array}
$$

\section{Experiment and analysis}

\subsection{Effect of SRR with different size of dictionary}

Experiment and test process are as follows:

Step 1. Set dictionary training parameters. The dictionary size is $1024, \lambda=0.06$, The image patches is $5 \times 5$, The number of sampled image blocks is 100,000 , The scaling factor is 2 。

Step 2. Acquisition of training samples image. training samples are obtained on the basis of remote sensing image degradation model. LRIs are interpolated and magnified using bicubic interpolation to the size of HRI.

Step 3. Dictionary training. HRI and LRIs are blocked size of $5 \times 5$, and then improved feature extract operator is used. Image training block is trained by the algorithm in ref[8]. $D_{h}$ and $D_{l}$.are obtained.

Step 4. Remote sensing image SRR. Reconstruction parameters are set respectively. Sparse regularization parameter is 0.06 , maximum number of overlaps between image blocks is 4 , scale factor is 2 , number of iterations of the back projection algorithm is 30 .

In the reconstruction process, the LRI with the original high-resolution test image is down-sampled 2 times and reconstruction image with high resolution are obtained by using bicubic interpolation, sparse representation in Ref[8] and our methods respectively.

This paper selects the root mean square error (RMSE) and peak signal-to-noise ratio (PSNR) to objectively evaluate the reconstructed image quality. The specific formula is as follows:

$$
\begin{gathered}
R M S E=\sqrt{\frac{\sum_{i=1}^{M} \sum_{j=1}^{N}\left(f_{i j}-f_{i j}^{\prime}\right)^{2}}{M \times N}} \\
P S N R=10 \times \lg \frac{255^{2}}{R M S E^{2}}
\end{gathered}
$$

where, $f_{i j}$ is the original HR image, $f_{i j}^{\prime}$ is the SRR result image, $M 、 N$ is the number of rows and columns of the image respectively. The smaller RMSE is, the larger PSNR is, and the better remote sensing image SRR result has.

The trained dictionary is shown in Figure 2. The effects of dictionary size to the results of SRR remote sensing images are tested, dictionary size are selected size of 128, 256, 512, 1024, 1536 and 2048 respectively. PSNR is compared in Figure 3, SRR results are compared in Figure 4.
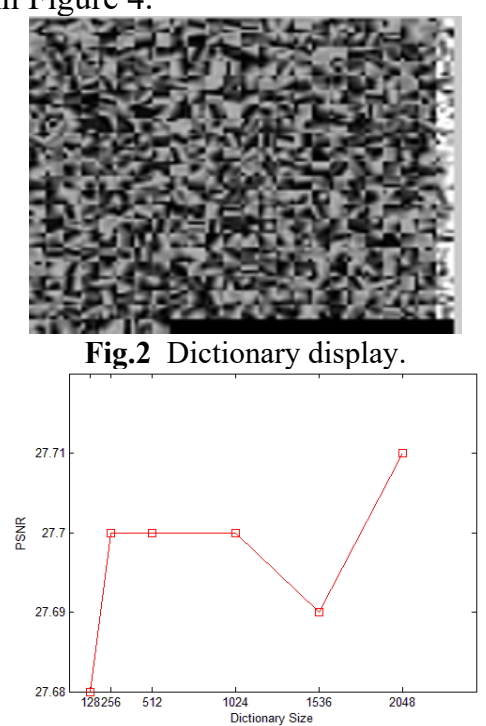

Fig.3 PSNR of remote sensing image SRR with different dictionary sizes

As can be seen from Fig.3, the larger size dictionary is, the larger PSNR is, and the better SRR result is. However, the larger size dictionary is, the longer SRR time cost. So the balance between the dictionary size and reconstruction time-cost should be considered.

\subsection{Compared results of SRR with different algorithms}

In this paper, 10 groups remote sensing are tested in experiments by using bicubic interpolation, sparse representation in Ref[8] and our methods respectively. Limited by paper length, 2 groups compared test results are shown in Fig.4 and Fig.5. Objective parameters RMSE and PSNR are compared in Table 1. 


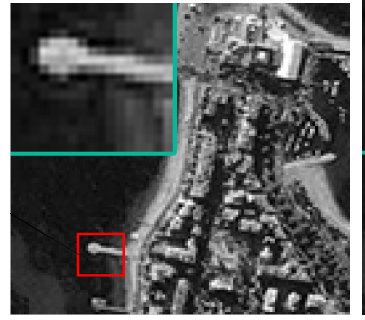

(a) low image

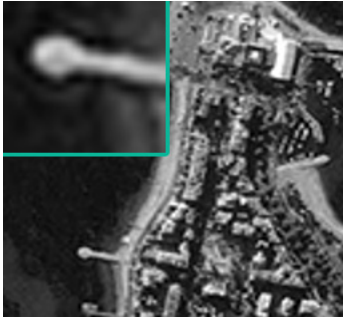

(b) result of bicubic interpolation (c) result of Ref[8]
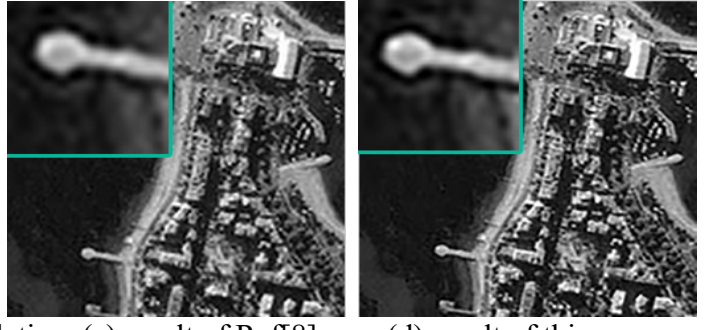

(d) result of this paper

Fig.4 Comparison result of the first group remote sensing image SRR

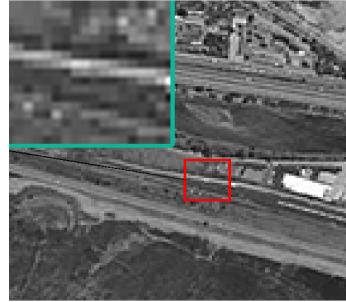

(a) low image

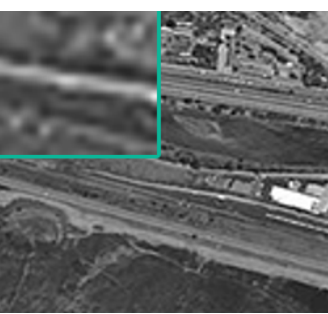

(b) result of bicubic interpolation

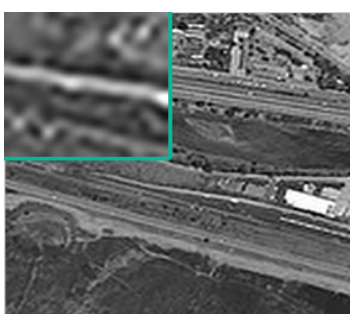

(c) result of Ref[8]

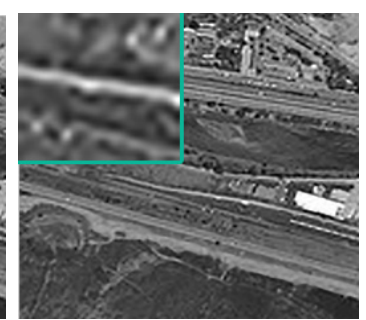

(d) result of this paper

Fig.5 Comparison result of the second group remote sensing image SRR

Tab.1 RMSE and PSNR comparison of test remote sensing image with different algorithms.

\begin{tabular}{ccccccc}
\hline Test images & \multicolumn{2}{c}{ Bicubic interpolation } & \multicolumn{2}{c}{ Algorithm in Ref[8] } & \multicolumn{2}{c}{ Our Method } \\
\cline { 2 - 6 } & RMSE & PSNR(dB) & RMSE & PSNR(dB) & RMSE & PSNR(dB) \\
\hline Group 1 & 16.16 & 23.96 & 14.02 & 25.19 & 13.78 & 25.35 \\
Group 2 & 16.93 & 23.56 & 13.59 & 25.47 & 13.35 & 25.62 \\
& & & & & & \\
\hline
\end{tabular}

Test experiment results show that the subjective visual effect of the proposed algorithm is improved, and more details information are occurred. Objective parameters are increased and PSNR is increased about $2.50 \mathrm{~dB}$ and $0.50 \mathrm{~dB}$, RMSE is decreased about 2.80 and 0.3 compared with bicubic interpolation algorithm and Ref[8] algorithm respectively.

\section{Conclusion}

Improved feature extraction operator is proposed in this paper and which is used in the field of remote sensing image sparse representation SRR. The experiment results show that the proposed algorithm improves remote sensing images visual effect and detail information significantly. Objective parameters PSNR is increased $0.50 \mathrm{~dB}$, RMSE is decreased 0.3 compared with sparse representation algorithm in Ref[8].

\section{Acknowledgment}

This work is supported the National Natural Science Foundation China (61601174), the Postdoctoral Research Foundation of Heilongjiang Province (LBHQ17150), the Science and Technology Innovative Research Team in Higher Educational Institutions of Heilongjiang Province (No. 2012TD007), the Fundamental Research Funds for the Heilongjiang Provincial Universities (KJCXZD201703) and the Science Foundation of Heilongjiang Province of China
(F2018026) .

\section{References}

1. Su H, Zhou J, Zhang Z H. Survey of superresolution image reconstruction methods. J. Acta Automatica Sin. 39, 3 (2013)

2. Zhang H, Zhang L, Shen H. A blind superresolution reconstruction method considering image registration errors. J. Int J Fuzzy Syst, 17, 11 (2015)

3. Zhao SR, Liu ZH, Liang $H$, et al. A mixed nonlocal prior model for image super-resolution reconstruction. J. Chinese J Electron, 26, 5 (2017)

4. $\mathrm{Pu} \mathrm{J}$, Zhang JP, Huang $\mathrm{H}$. A review of superresolution algorithms. J. Shandong University. 39, 7 (2009)

5. W. T. Freeman, T. R. Jones, E. C. Pasztor. Example based super-resolution. J. Comp. Graph. 22, 9 (2002)

6. Liu C, Shum H Y., Zhang C S. Two-step approach hallucinating faces: global parameters model and nonparametric model (CVPR, Kauai, USA, 2001)

7. Chang H, Yeung D Y. Super-resolution though neighbor embedding (CVPR, DC, USA, 2004)

8. Yang J, Wright J, Huang TS, et al. Image superresolution via sparse representation. J. IEEE Trans. On Image Processiong. 19, 12 (2010)

9. Ge G Z, Yang M. Single image super-resolution reconstruction based on sparse representation. Computer Technology \&Development 23, 3 (2013) 
10. Donoho D L. For most large under determined systems of linear equations, the minimum 1l-norm solution is also the sparsest solution. J. Commun Pur Appl. Math. 59, 32 (2006)

11. Lee H, Battle A, Raina R, et al. Efficient sparse coding algorithms (ANIPS, Paris, 2006)

12. Zhang Z, Li F Z, Zhao M B, et al. Robust neighborhood preserving projection by nuclear/12,1norm regularization for image feature extraction. IEEE Tans. On Image Processing, 26, 5 (2017) 